\title{
Features of examination of the ocular fundus of the Phoca sibirica
}

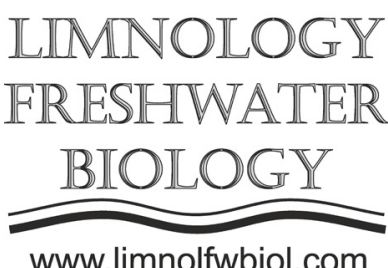

\author{
Karpova E.A. \\ Irkutsk State Agricultural University named after A.A. Ezhevsky, Timiryazeva Str. 59 Irkutsk, 664007, Russia
}

\begin{abstract}
The research purpose is to study the possibility of applying Tropicamide $1 \%$ for examining the fundus of the Baikal seal. Unlike terrestrial mammals, in marine mammals, the use of mydriatics does not cause required pupil expansion. This is due to the peculiarity of location of the pupil dilatator muscle. It is located perpendicular to the sphincter muscle and more closely adjacent to the iris base. Moreover, the dilator expands posteriorly over the enlarged base of each ciliary process. Tropicamide $1 \%$ causes small mydriasis (approximately $1 / 3$ of the possible maximum pupil expansion), which is enough to examine the central part of the fundus, but not enough to examine its periphery. Four-fold drug instillation with an interval of 15 minutes in absolute darkness is required to achieve persistent mydriasis. The optic disc is round. There are 8-9 retinal vessels that flow into the optic nerve. Unlike dogs and cats, the Baikal seal has arteries and veins of the retina of identical diameters. A reflective plate (tapetum lucidum) is visible. Its color varies from yellow-green to blue and gray depending on the age.
\end{abstract}

Keywords: Baikal seal, pupil, Tropicamide 1\%, mydriasis, fundus

\section{Introduction}

Unlike most terrestrial mammals, in pinnipeds the use of mydriatics does not cause required pupil expansion. This is due to the peculiarities of the anatomical structure of the pupil dilator, which differs from that of terrestrial mammals. The dilatator muscle is perpendicular to the sphincter muscle and more closely adjacent to the iris base. Moreover, the dilator expands posteriorly over the enlarged base of each ciliary process (Miller et al., 2010).

Subconjunctival administration of atropine causes partial expansion of the pupil. For example, in the fur seal, the duration is up to 4 days (Barnes and Smith, 2004). 1: 1000 epinephrine without preservatives introduced into the anterior eye chamber causes a better pupil dilatation (up to10 $\mathrm{mm}$ ) which is three parts of the dilated pupil in dogs (Colitz et al., 2010).

\section{Material and methods}

The research object was seals from the sealarium of Irkutsk region. The following research methods were employed: application of $1 \%$ midriacil (Tropicamide 1\%); fundus ophthalmoscopy with a PanOptic panoramic ophthalmoscope; photographic recording.

\section{Results}

Exposed to bright light, the pupil of the Baikal seal narrows. Its size becomes no more than $1 \mathrm{~mm}$ in width. With such myosis, it is impossible to perform ophthalmoscopy. Single instillation of Tropicamide 1\% (a drug used in humanitarian and veterinary medicine to achieve mydriasis by blocking the m-cholinergic receptors of the iris and ciliary muscle, thereby causing mydriasis) for 15 minutes did not expand the pupil to perform ophthalmoscopy. The pupil has a triangular shape with a base located dorsally and an apex located ventrally. After the third instillation of midriacyl 1\% and 30 minutes' wait, the pupil began to resemble a vertically located oval with a ventral narrowing, 6-7 $\mathrm{mm}$ wide. Further instillation did not allow us to achieve greater mydriasis, although in the dark pool, the pupil expands completely. In adult (7-18 years old) seals, the pupil has an oval shape, $9 \mathrm{~mm}$ in size. In 2-3 day-old animals, it was of a round shape with a diameter of $7 \mathrm{~mm}$. This expansion allows us to examine the central part of the fundus and the optic disc, but the periphery is difficult to visualize.

With ophthalmoscopy of the central part, the retinal vessels are well visualized. The optic disc is round. There are 8-9 retinal vessels that flow into the optic nerve. Unlike dogs and cats, in the Baikal seal, diameters of the arteries and veins of the retina are identical. A reflective plate (tapetum lucidum) is clearly 
visible. Its color varies from yellow green to blue and gray depending on the age.

\section{Coclusion}

The use of Tropicamide 1\% for examining the fundus is possible; 3-4-fold instillation of drops with an interval of 15 minutes is required to achieve persistent mydriasis. The resulting pupil dilatation is sufficient to examine the central part of the fundus, but it is impossible to examine the fundus periphery.

\section{References}

Barnes J.A., Smith J.S. 2004. Bilateral phacofragmentation in a New Zealand fur seal (Arctocehalus forsteri). Journal of Zoo and Wildlife Medicine 35: 110-112. DOI: 10.1638/02-037

Colitz C.M.H., Saville W.J.A., Renner M.S. et al. 2010. Risk factors associated with cataracts and lens luxations in captive pinnipeds in the United States and the Bahamas. Journal of the American Veterinary Medical Association 237: 429-436. DOI: 10.2460/javma.237.4.429

Miller S.N., Colitz C.M.H., Dubielzig R.R. 2010. Anatomy of the California sea lion globe. Veterinary Ophthalmology 13: 63-71. DOI: 10.1111/j.1463-5224.2010.00815.x 